\title{
A Continuum Model with Differential Evolution on Binary Phase Diagram of Ionic Surfactant Aqueous Solution ${ }^{\dagger}$
}

\author{
Yu Shi and Tom Beck* \\ Department of Chemistry, University of Cincinnati \\ E-mail: shiy4@ucmail.uc.edu \\ Phone: 5135564886
}

\begin{abstract}
The cell model had been shown to describe with reasonable accuracy the phase equilibrium properties for ionic amphiphile-water systems. Basing on the cell model, a continuum thermodynamic model is developed from three perspectives. First, incorporate the Helfrich free energy as amphiphilic molecules aggregate surface free energy; Second, modify the Poisson-Boltzmann equation by introducing the ion-specific dispersion interaction energy of the counter-ion in aqueous region with the aggregate surface to obtain the concentration distributions of both the amphiphile monomer and the counter-ions; Third, include the temperature dependence of chemical potential for the standard state transition, allowing for calculations on the binary phase diagram of a series of potassium carboxylate as well as of sodium carboxylate soaps. The differential evolution algorithm is applied to obtain the global minimum of the required criteria, including the boundary conditions of the electrostatic potential, the optimization of
\end{abstract}

\footnotetext{
${ }^{\dagger}$ A footnote for the title
} 
aggregate size with respect to the total free energy and the equilibrium of monomers transferring between the aggregate and aqueous region. The specific-ion effect are presented in the aggregate surface tensions and in the counter-ions distribution within the aqueous regions. The continuum model gives good agreement with the dimension sizes and phase boundaries (lamellar-cylindrical and cylindrical-micellar) which are determined with thermodynamic measurements.

\section{Introduction}

The duality of the amphiphilic molecules, arising from both the hydrophilic (polar) head group and the hydrophobic (apolar) tail group, endows these molecular self-assembly in the dilute solution with a diversity of aggregation geometries. The aggregation decreases the system free energy by reducing the contact of the apolar tail group with water molecules, while the polar head group being hydrated. ${ }^{1,2}$ Depending on the amphiphilic molecule types and solution conditions (e.g. concentration and temperature), the aggregate may be spherical (or globular, rodlike) micelles $\left(\mathrm{L}_{1}\right)$, cylinder $(\mathrm{E})$, planar bilayers $(\mathrm{D})$ and vesicles, etc. For the spherical micelles of ionic amphiphile, such as sodium dodecyl sulfate (SDS), Wennerstroms proposed the cell model, ${ }^{3}$ within which the aqueous solution is divided into spherical cells consisting of an aggregate and an aqueous region around. The free energy $G$ for the finite cell is expressed explicitly as summation of the standard state chemical potential, the aggregate surface free energy, the electrostatic interaction energy and the entropy energy in the aqueous region. The chemical potential of each component (water,amphiphile monomer and counterion) is derived directly through $\mu=\partial G / \partial n$, where $n$ is the number of each component in

the cell. Modeling both the monomer and the counter-ion as point-charge, the electrostatic interactions in the aqueous region are described on the basis of the non-linear PoissonBoltzmann equation. The activities of the both species calculated by the cell model are in good agreement with experimental findings. At higher amphiphile concentrations, the cylinder and planar bilayer aggregates begin to appear in sequence. The cell model had 
been developed to quantitatively describe the dimension sizes of all geometries, micellarcylindrical phase equilibrium and the cylindrical-lamellar phase equilibrium at $86^{\circ} \mathrm{C}$ for a series potassium carboxylate soaps. It was later advanced to reproduce the primary features of the ternary phase diagram of water-potassium decanoate- octanol $\left(\mathrm{H}_{2} \mathrm{O}_{-} \mathrm{C}_{9} \mathrm{H}_{19} \mathrm{COO}^{-} \mathrm{K}^{+}-\right.$ $\left.\mathrm{C}_{8} \mathrm{H}_{17} \mathrm{OH}\right)$ solution at $25^{\circ} \mathrm{C}$.

In spite of these descriptions with reasonable accuracy, the specific-ion effect and temperature impacts on phase behaviour are not included explicitly. The present work attempts to improve the cell model at three aspects. The first is to incorporate the Helfirch surface free energy ${ }^{4-6}$ so that the surface tension is given explicitly as a function of the surface curvatures rather than a constant $\gamma_{1}=18 \mathrm{~mJ} / \mathrm{m}^{2}$ for all aggregate geometries. The second important progress is to include the ion-specific dispersion. The expression of the dispersion energy between the hydrated counter-ion and the aggregate surface ${ }^{7-9}$ had been incorporated into the Poisson-Boltzmann equation for the spherical micelle in the work by Lukanov and Firoozabadi ${ }^{10}$. The modified Poisson-Boltzamnn (mPB) equation was solved for an infinite system, where both the electrostatic potential and electric field vanish far away from the micelle aggregate surface. It had been shown that mPB model ( without any adjustable parameters) gives reasonable agreements with the measured variations along with salt concentrations for the critical micelle concentration (CMC), aggregation number and micelle aggregate surface electrostatic potential. The mPB model also indicated that the Stern layers of steric exclusion or distances of closest approach are not essential to be imposed artificially when dispersion interaction are included. The third effort is to introduce an explicit temperature dependence to calculate the phase equilibria over a range of temperatures. The absence of the temperature dependence in the cell model leads to the inadequate description of the phase equilibria of palmitate potassium. ${ }^{11}$ In the earlier works ${ }^{12,13}$ it was assumed that the apolar hydrocarbon core of the aggregates is of fully fluid character and hence the standard state chemical potential of monomer in the aggregate $\mu_{m o n}^{0, a}$ is independent of aggregate geometries. The standard state chemical potential of monomer within the aqueous region 
$\mu_{m o n}^{0, w}$ and that of the counter-ion $\mu_{i o n}^{0, w}$ and the water molecule $\mu_{w a t}^{0}$ are also assumed to be independent of both temperature and concentration. Jonsson and Wennerstrom calculated $\Delta \mu_{\text {mon }}^{0}=\mu_{\text {mon }}^{0, w}-\mu_{\text {mon }}^{0, a}$ in an infinite micelle system at the experimental critical micellar concentration (CMC) for a series of alkyl with a particular head group. ${ }^{3,13}$ They employed Loeb $^{14}$ and Stigter ${ }^{15,16}$ analysis on the electrostatic effects to solve the Poisson-Boltzmann equation and then obtained the electrostatic free energy in the system $G_{e l}$. By assuming $2 \%$ of the monomers are in micellar aggregate ( with the aggregate number $n_{\text {agg }}$ ) they obtained the $\Delta \mu_{\text {mon }}^{0}=k T \ln \left(c_{\text {mon }}\right)-k T \ln \left(c_{\text {agg }} / n_{\text {agg }}\right)-G_{e l} / n_{\text {agg }}$, where the monomer concentration in aqueous region $c_{m o n}=0.98 \mathrm{CMC}$, and micelle concentration $c_{a g g}=0.02 \mathrm{CMC} / n_{\text {agg }}$. They applied this $\Delta \mu_{m o n}^{0}$ to all aggregate geometries at various concentrations and over a range of temperatures. This consistency of $\Delta \mu_{m o n}^{0}$ is contrary to the conclusions from the investigation by Gruen, ${ }^{17,18}$ Ben-Shaul and Gelbart, ${ }^{19-22}$ Ruckenstein ${ }^{23}$ and Nagarajan. ${ }^{24}$ As Nagarajan pointed out the tails inside the apolar core are not in a state identical to that in the hydrocarbon liquid, since one end of the tail is constrained to the aggregate surface while the entire chain has to maintain a uniform density equal to that of hydrocarbon liquid in the apolar core. Explicit expressions of free energy for conformational constraint were given by Nagarajan ${ }^{24}$ as $\Delta \mu_{\text {mon }}^{0, L_{1}}=(27 / 8) v_{\text {tail }} L_{\text {grid }} / a_{0}^{2}, \Delta \mu_{\text {mon }}^{0, E}=(20 / 8) v_{\text {tail }} L_{\text {grid }} / a_{0}^{2}$, $\Delta \mu_{\text {mon }}^{0, D}=(10 / 8) v_{\text {tail }} L_{\text {grid }} / a_{0}^{2}$, where $L_{\text {grid }}=4.6 \AA v_{\text {tail }}$ is the volume of surfactant tail, $\mathrm{a}_{0}$ is the apolar core surface area per tail.

In addition to the temperature dependence of water dielectric constant, density, and that of the apolar group volume and transition free energy from water into aggregates, we introduce the temperature dependence for both $\delta \mu_{a m p}^{0,2}=\Delta \mu_{a m p}^{0, E}-\Delta \mu_{a m p}^{0, D}$ and $\delta \mu_{a m p}^{0,3}=$ $\Delta \mu_{a m p}^{0, L_{1}}-\Delta \mu_{a m p}^{0, E}$. The difference of standard state chemical potentials of water $\left(\delta \mu_{w a t}^{0,2}\right.$ and $\left.\delta \mu_{\text {wat }}^{0,3}\right)$ are considered as well. Due to the lack of experiment measurements, herein temperature dependencies are obtained by fitting the both $\mathrm{D} / \mathrm{E}$ phases boundaries and $\mathrm{E} / \mathrm{L}_{1}$ phase boundaries at three different temperatures to the experimental binary phase diagram for a series of potassium and sodium carboxylate soaps. 
In the cell model, to solve the PB equations, a good initial guess for the aggregate size $b$ and both the amphiphile monomer concentration $c_{m o n}^{0}$ and the counter-ion concentration $c_{i o n}^{0}$ at the cell boundary are required to avoid divergence. ${ }^{3}$ Once the convergence for the PB equations as well as boundary conditions are achieved, we need to check if $b$ is optimal and if the monomer is in equilibrium between the aggregate and aqueous region, $\mu_{m o n}^{a}=\mu_{m o n}^{w}$. In previous works, ${ }^{3}$ the New-Raphson minimization algorithm is used to obtain the convergence from a rather good initial guesses for aggregate size and concentrations at the cell boundary. In the present work, we used the differential evolution algorithm to obtain the minimum of the sum of squares of the boundary conditions of electrostatic potential, optimization of aggregate size with respect to the total free energy $G$ and the monomer equilibrium of monomers between the aggregate and the aqueous region. As a stochastic method (contrary to the gradient methods) to find the global minimum, differential evolution ${ }^{25}$ can search large areas of parameter space, avoiding the requirement of good initial guess.

\section{Continuum Model}

We develop a continuum model in the framework of the cell model, where the ionic surfactant aqueous solution is divided into cells of the same size. Each cell comprises an aggregate at the center, a surrounding aqueous region and an interface between them. ${ }^{3,13}$ The cell and the aggregate share the same shape. As for the series of carboxylate soaps, we investigated the equilibrium properties of three phases where the cell is of one of three geometries, lamelle (planar bilayers aggregate), cylinder (cylindrical aggregate) and micelle (spherical aggregate). In one particular phase, all of the aggregation are assume to share the same dimension sizes, while the distribution of the aggregate sizes ${ }^{13}$ will not be discussed herein. Waters and counter-ions reside only in the aqueous region. The monomers transfer between the aqueous region and the aggregate. The aggregate core consists of apolar tails and is assumed to be of fully hydrocarbon liquid. ${ }^{23,26}$ The interface carrying the charges of the head group is 
approximated as a continuum elastic surface by neglecting the porous feature. ${ }^{3}$ The total Gibbs free energy is expressed as

$$
\begin{aligned}
G & =n_{\text {mon }}^{a} \mu_{m o n}^{0, a}+n_{m o n}^{w} \mu_{m o n}^{0, w}+n_{i o n}^{w} \mu_{i o n}^{0, w}+n_{w a t}^{w} \mu_{w a t}^{w} \\
& +E_{e l}+E_{\text {disp }}+G_{m i x}+G_{A}
\end{aligned}
$$

here $n_{m o n}^{a}$ and $\mu_{m o n}^{0, a}$ are the number of monomer in the aggregate and the monomer standard state chemical potential; $n_{m o n}^{w}$ and $n_{i o n}^{w}$ are the monomer number and the counter-ion number in the aqueous region; $\mu_{m o n}^{0, w}$ and $\mu_{i o n}^{0, w}$ are the standard state chemical potentials, correspondingly; $n_{w a t}^{w}$ and $\mu_{w a t}^{w}$ are the water number and water chemical potential. $E_{e l}$ is the electrostatic energy of the charged components in the aqueous region, and $E_{\text {disp }}$ the dispersion energy of the counter-ions with the charged hydrocarbon surface. $G_{m i x}$ is the free energy contribution from ideal mixing of components in the aqueous region, and $G_{A}$ is the aggregate surface free energy. The electrostatic energy $E_{e l}$ and dispersion energy $E_{\text {disp }}$ can be treated with the following $\mathrm{mPB}$ equation

$$
\nabla^{2} \phi=-\frac{F}{\epsilon_{r} \epsilon_{0}}\left\{c_{i o n}^{0} \exp \left(-\frac{e \phi(r)+U_{\text {disp }}(r)}{k T}\right)+c_{\text {mon }}^{0} \exp \left(\frac{e \phi(r)}{k T}\right)\right\}
$$

where $F$ is the Faraday contant, $\epsilon_{0}$ the vacuum permittivity, $\epsilon_{r}$ water dielectric constant (relative permitivity), $e$ the unit charge, and $k T$ the Boltzmann factor, $c_{i o n}^{0}$ and $c_{m o n}^{0}$ the counter-ion and monomer concentrations at the cell boundary where $\phi=0$ by convention. $\phi(r)$ and $U_{d i s p}(r)$ are the electrostatic and dispersion potentials. When only two adjacent aggregates are considered, we have $U_{\text {disp }}=0$ at the cell boundary, resulting in the net dispersion potential as

$$
U_{\text {disp }}(r)=\frac{B}{r^{3}} h(r)-\frac{B}{(2 L-r)^{3}} h(2 L-r)
$$


where $L$ is the size the aqueous region and the interaction parameters $B$ are the same as listed in previous work ${ }^{10}$ and

$$
h(r)=1+\frac{2 r}{\sqrt{\pi} a_{\text {ion }}}\left[\frac{2 r^{2}}{a_{i o n}^{2}}-1\right] \exp \left(-\frac{r^{2}}{a_{i o n}^{2}}\right)-\left[1+\frac{4 r^{4}}{a_{i o n}^{4}}\right]\left(\frac{r}{a_{i o n}}\right)
$$

where $a_{\text {ion }}$ Gaussian radius of the ion. ${ }^{10}$ The electrostatic energy is then

$$
E_{e l}=\frac{1}{2} n_{m o n}^{a}(-e) \phi_{A}+\frac{F f_{d}}{2} \int_{b}^{b+L}\left(c_{i o n}-c_{m o n}\right) \phi r^{d-1} d r
$$

and dispersion energy is

$$
E_{\text {disp }}=N_{A} f_{d} \int_{b}^{b+L} c_{i o n} U_{d i s p} r^{d-1} d r
$$

and the ideal mixing free energy in the aqueous bulk is

$$
G_{m i x}=-T S_{m i x}=R T f_{d} \int_{b}^{b+L}\left\{c_{\text {ion }}\left[\ln \left(c_{\text {ion }} / c_{0}\right)-1\right]+c_{m o n}\left[\ln \left(c_{m o n} / c_{0}\right)-1\right]\right\} r^{d-1} d r
$$

where $f_{d}=A / b^{d-1}, A$ is the aggregate surface, $\phi_{A}$ is the electrostatic potential at the aggregate surface, $N_{A}$ Avogadro constant, $b$ the aggregate size, $c_{0}$ the total concentration constant $(55.5 \mathrm{M}), d$ the dimensionality, 1,2,3 for lamellar,cylindrical and spherical system, respectively.

We assume that the charged head groups reside on the surface with a constant charge density $\sigma$. And then there are primarily two contributions to $G_{A}$, the first one is the repulsion due to the contact of apolar tails with waters; the second one is the repulsion arising from the steric and electrostatic interactions between head groups. Herein we regard the aggregate as a macroscopic system ${ }^{12}$ so that the aggregate surface is assumed to be an elastic continuum for which the surface tension is given by the Helfrich free energy ${ }^{4,5}$

$$
\gamma=\gamma_{0}+2 \kappa\left(H-H_{0}\right)^{2}+\bar{\kappa} H_{g}
$$


where $H=\frac{1}{2}\left(c_{1}+c_{2}\right)$ and the Gaussian curvature $H_{g}=c_{1} c_{2}, \gamma_{0}$ is the intrinsic surface tension, $c_{1}$ and $c_{2}$ are the two principal curvatures, $H_{0}$ is the spontaneous curvature, $\kappa$ is the bending constant, $\bar{\kappa}$ is the splay constant. For micellar aggregate, we have $c_{1}=c_{2}=1 / b$ and $\bar{\kappa}=2 \kappa\left(H_{0} b-1\right)$; for cylindrical aggregate, $c_{1}=0 ; c_{2}=1 / b$; for lamellar aggregate, $c_{1}=c_{2}=0$ and $\gamma_{1}=\gamma_{0}+2 \kappa H_{0}^{2}$. In the isotropic micellar solution there is an additional contribution from the ideal mixing entropy of spherical aggregates in the solution,

$$
G_{m i x}^{m i c}=k T\left[\ln \left(c_{m} / c_{0}\right)-1\right]
$$

where $c_{m}$ is the mole fraction of micelles in the solution. Substituing the concentrations of monomers and counter-ions

$$
\begin{aligned}
& c_{\text {ion }}=c_{\text {ion }}^{0} \exp \left(-\frac{e \phi(r)+U_{\text {disp }}(r)}{k T}\right) \\
& c_{\text {mon }}=c_{\text {mon }}^{0} \exp \left(\frac{e \phi(r)}{k T}\right)
\end{aligned}
$$

into the above expressions, we obtain the total free energy. The detailed derivation is given in the Appendix A.

$$
\begin{aligned}
G= & n_{\text {mon }}^{a} \mu_{\text {mon }}^{0, a}+n_{\text {mon }}^{w} \mu_{\text {mon }}^{0, w}+n_{\text {ion }}^{w} \mu_{\text {ion }}^{0, w}+n_{\text {wat }}^{w} \mu_{\text {wat }}^{w}+\gamma A \\
& +n_{\text {mon }}^{a}(-e) \phi_{A}-E_{\text {el }}+k T\left[n_{\text {ion }}^{w} \ln \left(c_{\text {ion }}^{0} / c_{0}\right)+n_{\text {mon }}^{w} \ln \left(c_{\text {mon }}^{0} / c_{0}\right)\right]-k T\left(n_{\text {ion }}^{w}+n_{m o n}^{w}\right)
\end{aligned}
$$

Taking derivative of the total free energy $G$ with respect to the molecular number gives us the chemical potentials for each component, 


$$
\begin{aligned}
& \mu_{w a t}^{w}=\mu_{w a t}^{0}-R T\left(c_{\text {ion }}^{0}+c_{\text {mon }}^{0}\right) v_{w a t} \\
& \mu_{i o n}^{w}=\mu_{i o n}^{0, w}+k T \ln c_{i o n}^{0}-R T c_{m o n}^{0} v_{i o n} \\
& \mu_{m o n}^{w}=\mu_{m o n}^{0, w}+k T \ln c_{m o n}^{0}-R T c_{i o n}^{0} v_{m o n} \\
& \mu_{\text {mon }}^{a}=\mu_{\text {mon }}^{0, a}+(-e) \phi_{A}+\frac{1}{n}\left\{\gamma A-E_{\text {el }}-k T\left(n_{\text {ion }}+n_{\text {mon }}\right)+R T V_{\text {wat }}\left(c_{\text {ion }}^{0}+c_{\text {mon }}^{0}\right)\right\}
\end{aligned}
$$

where $V_{\text {wat }}$ is the volume of aqueous region in each cell, $v_{\text {wat }}$ is the volume of water molecule, $v_{\text {ion }}$ counter ion volume, $v_{m o n}$ surfactant monomer volume.

\section{Computation}

The geometry properties of the amphiphile molecule are determined first. The amphiphile molecule volume $v_{a m p}$ and the water molecule volume $v_{w a t}$ are calculated from the specific volumes given in the work by Skoulios. ${ }^{27,28}$ The volume of counter-ion $v_{\text {ion }}$ are regarded as a hard sphere with radii $\mathrm{Na}^{+}\left(a_{i o n}=1.33 \AA\right)$ and $\mathrm{K}^{+}\left(a_{\text {ion }}=1.77 \AA\right)$ as proposed by Ninham. ${ }^{29,30}$ The volume of the monomer is $v_{m o n}=v_{a m p}-v_{i o n}$. The volume of tail group is calculated as the sum of volumes of methylene and methyl groups, $v_{t a i l}=v_{C H_{3}}+\left(n_{c}-1\right) v_{C H_{2}},{ }^{10}$ and

$$
\begin{aligned}
& v_{C_{H_{3}}}=54.6+0.1240(T-298) \AA^{3} \\
& v_{C H_{2}}=26.9+0.0146(T-298) \AA^{3}
\end{aligned}
$$

The extended length of the tail group is given by Tanford ${ }^{1,2}$ as $l_{\text {exd }}=1.5+1.265 n_{c}$, where $n_{c}$ is carbon number in the tail. The aggregate number $n_{\text {agg }}=V_{\text {agg }} / v_{\text {mon }}=V_{\text {core }} / v_{\text {tail }}$, so that the aggregate extended size is $b_{\text {exd }}=l_{\text {exd }}\left(\frac{v_{\text {mon }}}{v_{\text {tail }}}\right)^{1 / d}$ where $V_{\text {core }}$ is the apolar core of the aggregate.

Next in one particular phase, we have $\mu_{m o n}^{a}=\mu_{m o n}^{w}$ for the equilibrium of monomer between aggregate and aqueous region. Having expressed the head group contribution in 
the surface free energy, we use the free energy change of transferring the tail group from aqueous phase to a liquid hydrocarbon phase to account for the standard state chemical potential difference $\Delta \mu_{m o n}^{0}=\mu_{\text {mon }}^{0, w}-\mu_{\text {mon }}^{0, a}$, which is expressed as sum of contributions from both methylene group and methyl group, $\Delta \mu_{m o n}^{0}=\left(n_{c}-1\right) \Delta \mu_{C H_{2}}^{0}+\Delta \mu_{C H_{3}}^{0},{ }^{23,24}$

$$
\begin{aligned}
& \left(\Delta \mu_{C H_{2}}^{0}\right) / k T=5.85 \ln T+896 / T-36.15-0.005600 T \\
& \left(\Delta \mu_{C H_{3}}^{0}\right) / k T=3.38 \ln T+4064 / T-44.13+0.002596 T
\end{aligned}
$$

where the temperature dependence is explicitly included. The dependence of water dielectric constant $^{23}$ and density on temperature (from Dortmund Data Bank) is given as

$$
\begin{aligned}
\epsilon_{r} & =87.74 \exp (-0.0046(T-273)) \\
\rho_{w} & =0.14395 / 0.0112\left(1+(1-T / 649.727)^{0.05107}\right)
\end{aligned}
$$

Prior to solving the mPB equation Eq.[2], we have to know the aggregate size $b$, which is determined by the optimization $\left(\frac{\partial G}{\partial b}\right)_{n}=0$ (Gob). Combining with the Eq.[11], we get

$$
\left(\frac{\partial G}{\partial b}\right)_{n}=2 E_{e l}-\gamma A-A(d-1)\left[2 \kappa\left(H-H_{0}\right) / b+(d-2) \bar{\kappa} / b^{2}\right]
$$

and for micellar aggregate there is an extra term

$$
\left(\frac{\partial G_{\text {mix }}^{\text {mic }}}{\partial b}\right)_{n}=\frac{-3 k T}{b} \ln \left(c_{m} / c_{0}\right)
$$

Conventionally, at the cell boundary we have $\phi=0$ and $\nabla \phi=0$, while at the aggregate surface, we have

$$
\left(\nabla \phi \cdot n_{A}\right)_{A}=-\sigma / \epsilon_{r} \epsilon_{0}
$$

, where $n_{A}$ is unit vector normal to aggregate surface and the charge density $\sigma$ is constant over the surface. 
Finally, for an aggregation in an aqueous bulk region of size L, we have a system of three equations, monomer equilibrium, optimization of aggregate size, and electrostatic potential boundary condition at the aggregate surface. Employing the least-squares method gives us the solutions of the system of equations by minimizing the sum of squares of each equation. The solutions are the optimal aggregate size $b$, the concentration at aggregate surface of counter-ion $c_{i o n}^{0}$ and that of monomer $c_{m o n}^{0}$. We use the differential evolution (DE) $\operatorname{algorithm}^{25}$ to search the minimum of the sum of square within a large parameter space, comparing to that the Newton-Raphson algorithm requires a rather good initial guess to obtain the convergence which is not always easy to provide. Within each DE iteration, for a given $b$, solving the mPB through integration (by the ODEINT package in Scipy) would give both $c_{i o n}^{0}$ and $c_{m o n}^{0}$. Multiply hierarchical DE has been applied to the calculations for $\gamma_{1}, \gamma_{0}$ and $H_{0}$.

\section{Results And Discussion}

\section{Surface tension Calculation}
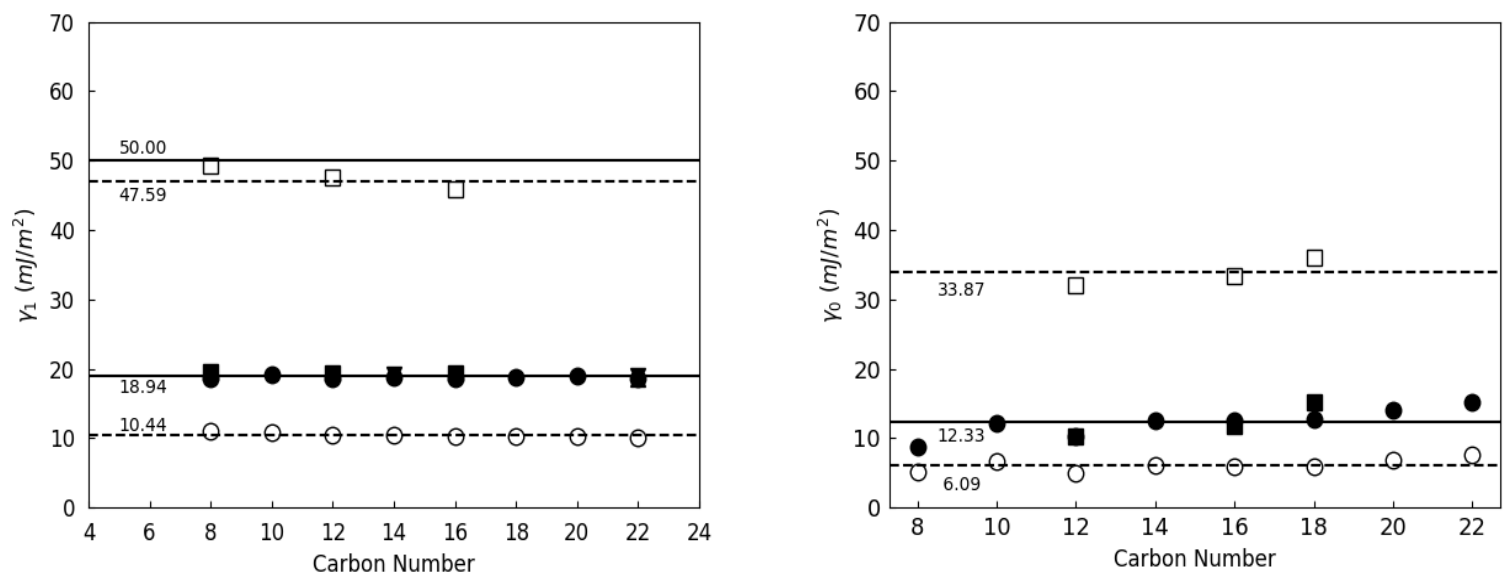

Figure 1: Left the lamellar aggregate surface tension $\gamma_{1}\left(\mathrm{~mJ} / \mathrm{m}^{2}\right)$; Right the intrinsic surface tension $\gamma_{0}\left(\mathrm{~mJ} / \mathrm{m}^{2}\right)$ calculated from the cylindrical aggregates. In both plots, we have $\mathrm{K}^{+}$ square, $\mathrm{Na}^{+}$circle; the solid symbols are for the systems only with electrostatic interaction, while the open symbols are systems of dispersion. 
Table 1: Lamella aggregate surface tension $\gamma_{1}$ with electrostatics only and with dispersion. Cylindrical aggregation intrinsic surface tension $\gamma_{0}$ with electrostatics only and with dispersion.

\begin{tabular}{cccccccccc}
\hline \hline Lamella & & 8 & 10 & 12 & 14 & 16 & 18 & 20 & 22 \\
\hline $\mathrm{Na}^{+}$Elect. & $\gamma_{1}$ & 19.60 & & 19.43 & & 19.31 & & & \\
$\mathrm{~K}^{+}$Elect. & $\gamma_{1}$ & 18.61 & 19.10 & 18.62 & 18.86 & 18.61 & 18.86 & 18.97 & 18.66 \\
\hline $\mathrm{Na}^{+}$Disp. & $\gamma_{1}$ & 49.23 & & 47.60 & & 45.93 & & & \\
$\mathrm{~K}^{+}$Disp. & $\gamma_{1}$ & 11.00 & 10.92 & 10.41 & 10.40 & 10.19 & 10.26 & 10.26 & 10.08 \\
\hline \hline Cylindrical & & 8 & 10 & 12 & 14 & 16 & 18 & 20 & 22 \\
\hline $\mathrm{Na}^{+}$Elect. & $\gamma_{0}$ & & & 10.36 & & 11.70 & 15.17 & & \\
$\mathrm{~K}^{+}$Elect. & $\gamma_{0}$ & 8.71 & 12.21 & 10.29 & 12.61 & 12.48 & 12.78 & 14.14 & 15.16 \\
\hline $\mathrm{Na}^{+}$Disp. & $\gamma_{0}$ & & & 32.13 & & 33.42 & 36.07 & & \\
$\mathrm{~K}^{+}$Disp. & $\gamma_{0}$ & 5.10 & 6.57 & 4.90 & 6.01 & 5.92 & 5.90 & 6.77 & 7.54 \\
\hline
\end{tabular}

The aggregate dimensions of series of potassium carboxylate soap and sodium carboxylate soap had been measured by low-angle X-ray diffraction ${ }^{27}$ at $86^{0} \mathrm{C}$, from which we calculated the aqueous bulk size $L$ and the aggregate size $b$. Substituting the two sizes into the continuum model, for a given surface tension $\gamma_{1}$, we calculated the sum of square of the Gob values for all the experimental sizes. Evolving the surface tension through the differential evolutionary generic algorithm, we obtained $\gamma_{1}$ which minimizes the sum of square. For example, as for the palmitate sodium lamellar aggregation with dispersion, we got $\gamma_{1}=45.93$ $\left(\mathrm{mJ} / \mathrm{m}^{2}\right)$ which gives a minimum 0.17 for 5 experimental sizes; for the palmitate potassium lamellar aggregation with dispersion, we got $\gamma_{1}=10.19\left(\mathrm{~mJ} / \mathrm{m}^{2}\right)$ with a minimum 0.02 over 7 sizes. We list all the surface tension values in Table 1 . When there is only electrostatic interaction, within a range of $0-70\left(10^{-3} \mathrm{~J} / \mathrm{m}^{2}\right)$, we get the average $\gamma_{1}$ value 18.94 with a deviation of $1.7 \%$ for all the carboxylate soap lamellar aggregations as shown in the Left panel of Fig.1. This is close to 18.00 in Wennerstrom works, ${ }^{3,12,13}$ where it was proposed as a constant for all aggregate geometries. In Parsegian work ${ }^{31}$ on transition between the lamellar and the cylindrical phases, the average value $18.50 \mathrm{~mJ} / \mathrm{m}^{2}$ with deviation of $5.0 \%$ was used for both geometries, which was obtained by fitting to experimental aggregation dimensions. The experimental sizes bring the ion specific effect into Parsegian's calculations, resulting in the surface tension of sodium soaps are slightly larger than that of the potassium soap with 
the same tail-group, correspondingly . The continuum model calculations with electrostatic interaction reproduced the difference, which is shown in Fig.1. However when we added the dispersion into the Poisson-Boltzmann equation, the difference between potassium soaps and sodium soaps are significant. The average $\gamma_{1}$ of sodium soaps is $47.59 \mathrm{~mJ} / \mathrm{m}^{2}$ with deviation $2.8 \%$, which is close to the value of $43.42 \mathrm{~mJ} / \mathrm{m}^{2}$ estimated for palmitate soap in the work by Ruckenstein ${ }^{23}$ where they assumed that the aggregate core-water interfacial tension is equal to that between aliphatic hydrocarbon of the same molecular weight as the surfactant tail and the surrounding waters. The average $\gamma_{1}$ of potassium soaps is of an average 10.44 $\mathrm{mJ} / \mathrm{m}^{2}$ with deviation $3.0 \%$. For a macroscopic interface between hydrocarbon liquid and water it is of the order of $\gamma_{1}=50 \mathrm{~mJ} / \mathrm{m}^{2}$, which is employed as the interface tension of ionic surfactant aggregate. ${ }^{10,32,33}$ Tanford, ${ }^{34}$ on a molecular level, proposed that the hydrocarbon core-water interface is shielded by either polar head-group or ions bound onto the interface reduces the residual contact and hence lowers the surface tension and estimate empirically an approximation of $25 \mathrm{~mJ} / \mathrm{m}^{2}$ for the residual water-hydrocarbon contact . Considering the carboxylate soaps having the same tail group, such as potassium palmitate C16K or sodium palmitate $\mathrm{C} 16 \mathrm{Na}$, it is the ion-specific dispersion that results in the large gap of $\gamma_{1}$ values between $\mathrm{Na}^{+}$and $\mathrm{K}^{+}$. In Fig. 2 we showed how the counter-ions distribute in the aqueous bulk ( $L=5.22 \AA$ ) at $86^{\circ} \mathrm{C}$ for the lamellar aggregations either with or without dispersion. For calculations with only electrostatic interaction, the ion-specific effect is primarily from the ion size $\left(\mathrm{Na}^{+}\right.$of radius $1.33 \AA$ and $\mathrm{K}^{+}$of radius $1.77 \AA$ which results in close properties, such as the close aggregate sizes, sodium palmitate $b=12.89 \AA$ and potassium palmitate $b$ $=12.92 \AA$ as well as close counter-ion distributions. However there are more $\mathrm{K}^{+}$attached to the lamellar aggregate surface ( $87 \%$ to $3.54 \AA$ ) and less $\mathrm{Na}^{+}$attached $(79 \%$ to $2.66 \AA$ ) as shown in the right panel of Fig. 2. This indicates that more residual contact of hydrocarbon core with waters in the $\mathrm{C} 16 \mathrm{Na}$ lamellar aggregation. Consequently surface tension of $\mathrm{C} 16 \mathrm{Na}$ lamellar aggregation $\left(\gamma_{1}=19.31 \mathrm{~mJ} / \mathrm{m}^{2}\right)$ is slight larger that of $\mathrm{C} 16 \mathrm{~K}\left(\gamma_{1}=18.61 \mathrm{~mJ} / \mathrm{m}^{2}\right)$, which is in agreement with calculation in the Parsegian work. ${ }^{31}$ Moreover when dispersion 
is added, both system have the same aggregate size $b=12.94 \AA$. The positive contributions to the surface tension from the interactions between head groups should be the same due to the same dimensions. The similar observations for the cylindrical aggregate are shown in the Fig.6 in Appendix B. Consequently the significant difference of $\gamma_{1}$ values between potassium palmitate and sodium palmitate should arise from the dispersion, which results in more $\mathrm{K}^{+}$attached to the lamellar aggregate surface $(92 \%)$ and less $\mathrm{Na}^{+}$attached $(68 \%)$, leading to more residual contact of the sodium palmitate aggregate and hence much large surface tension than that of potassium palmitate aggregate.
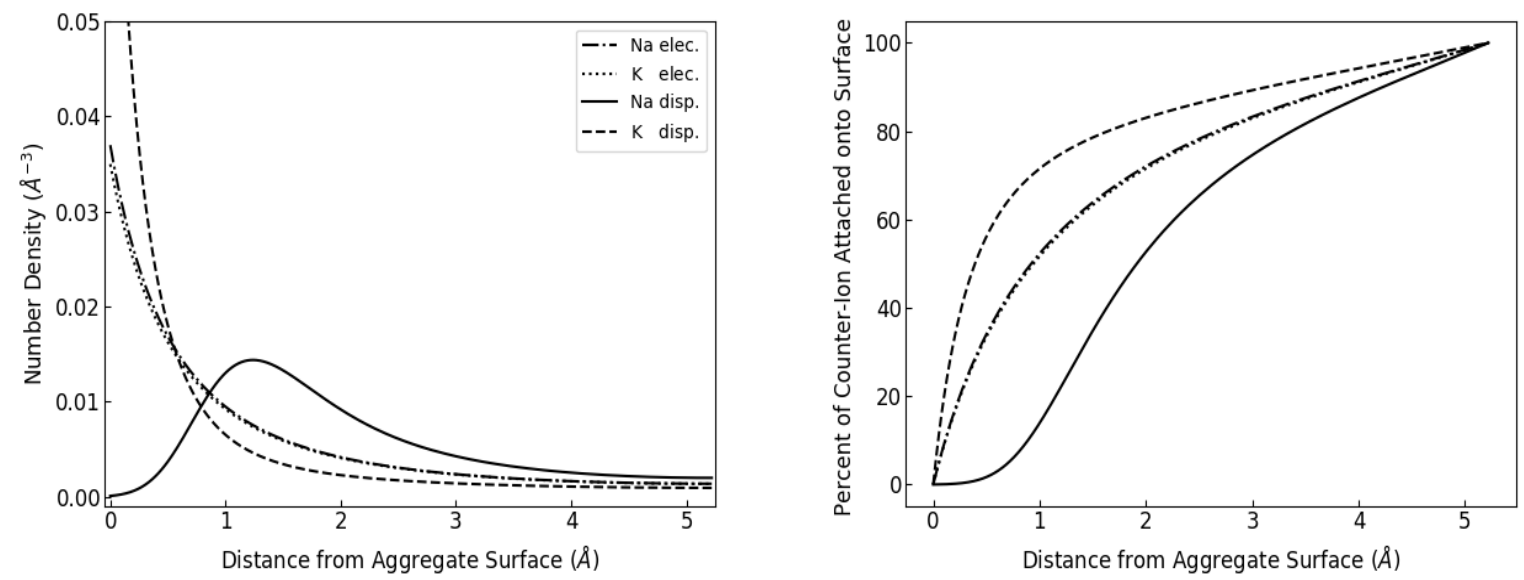

Figure 2: Left panel, the counter-ion number density in the aqueous region of $L=5.22 \AA$ of the palmitate soap lamellar aggregate systems. Right panel, the fraction of the counter-ion close to the lamellar aggregate surface. For both panels, the dash-dotted and dotted lines are for the $\mathrm{Na}^{+}$and $\mathrm{K}^{+}$only with electrostatic interaction, respectively; The solid and dashed lines are for the $\mathrm{Na}^{+}$and $\mathrm{K}^{+}$with dispersion, respectively.

As for the cylindrical aggregations, evolving the both intrinsic surface tension $\gamma_{0}$ and spontaneous curvature $H_{0}$ in the genetic algorithm, we obtained the their values by minimizing the sum of square of Gob. As shown in the right panel of Fig.1, the calculations with only electrostatic interaction between counter-ion and monomer give an average $\gamma_{0}=12.33$ $\mathrm{mJ} / \mathrm{m}^{2}$ with deviation $15.7 \%$; the calculations with dispersion generate large gap between average of sodium soaps $33.87 \mathrm{~mJ} / \mathrm{m}^{2}$ with deviation $4.8 \%$ and average of potassium soap $6.09 \mathrm{~mJ} / \mathrm{m}^{2}$ with deviation 13.3\%. In the left panel of Fig.6 (Appendix B), we show the 
counter-ion distributions in the aqueous bulk $L=8.86 \AA$. For calculations with only electrostatic interaction, the $\mathrm{C} 16 \mathrm{Na}$ aggregate size $b=18.14 \AA$, and the $\mathrm{C} 16 \mathrm{~K}$ aggregate size $b=18.13 \AA$ while calculation with dispersion give us $b=18.16 \AA$ for both cylindrical aggregates. In the right panel, we can see that to the diameter of counter-ions, the calculation with electrostatic interaction results in that $71 \% \mathrm{~K}^{+}$attached to the cylindrical aggregate surface and $64 \% \mathrm{Na}^{+}$attached,leading to close surface tension, $\mathrm{Na}^{+}\left(\gamma_{1}=18.97 \mathrm{~mJ} / \mathrm{m}^{2}\right)$ and $\mathrm{K}^{+}\left(\gamma_{1}=18.94 \mathrm{~mJ} / \mathrm{m}^{2}\right)$; while the calculation with dispersion generates that $80 \%$ of $\mathrm{K}^{+}$ attached to the aggregate surface, $47 \%$ of $\mathrm{Na}^{+}$and hence larger gap between surface tension of $\mathrm{Na}^{+}\left(\gamma_{1}=33.42 \mathrm{~mJ} / \mathrm{m}^{2}\right)$ and that of $\mathrm{K}^{+}\left(\gamma_{1}=6.10 \mathrm{~mJ} / \mathrm{m}^{2}\right)$. The spontaneous curvature $H_{0}$ and the bending constant $\kappa$ values are listed in Table.2 in the Appendix B.

\section{Standard States Chemical Potential Correction and Phase Diagram}

\section{Calculation}
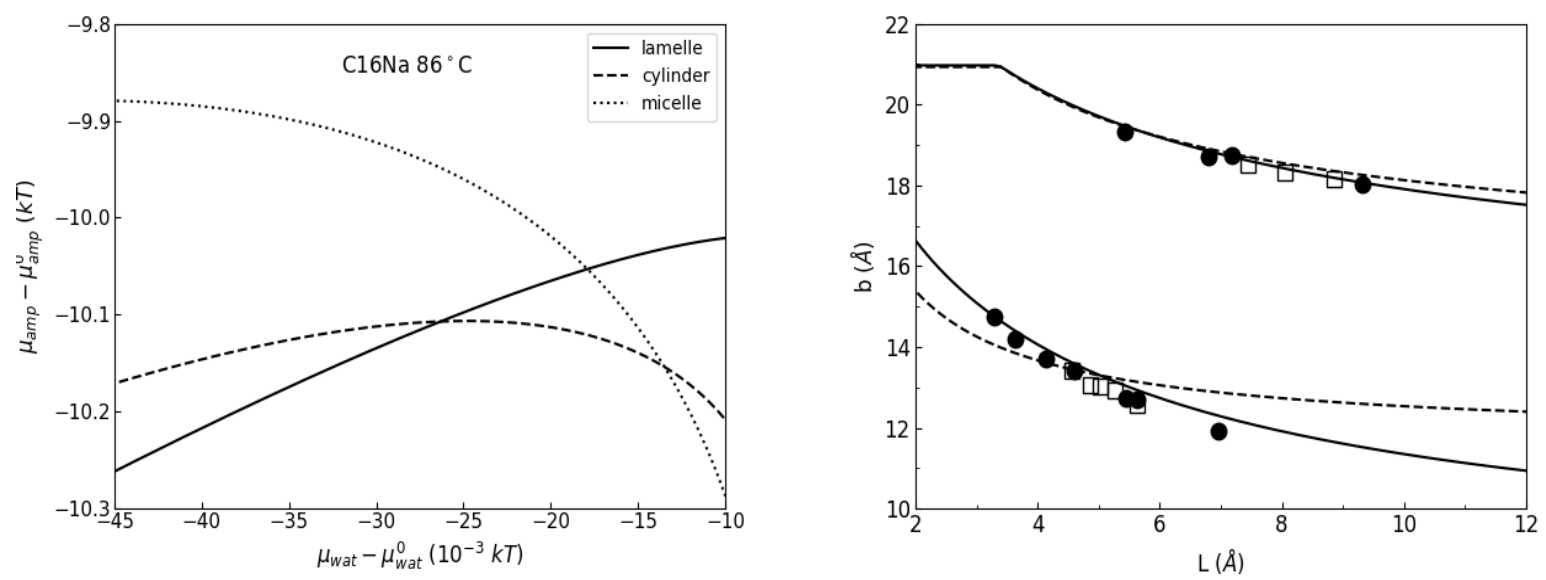

Figure 3: Left Panel,the chemical potentials of amphiphilic molecule $\mu_{a m p}$ and water molecules $\mu_{\text {wat }}$ in the sodium palmitate systems at $86{ }^{\circ} \mathrm{C}$. The solid line is for the lamellar aggregation, the dashed line for the cylindrical aggregation and dotted line for the micellar aggregation. Right panel,the aggregation dimensions (aqueous region size $L$ and aggregate size $b$ ) of the Palmitate soaps at $86{ }^{\circ} \mathrm{C}$. the open square is for counter-ion $\mathrm{Na}^{+}$, the solid circle is for the $\mathrm{K}^{+}$; the solid lines are calculated for $\mathrm{K}^{+}$and dashed lines are calculated for $\mathrm{Na}^{+}$. The upper lines are for the cylindrical aggregation cases and the lower lines are for the lamellar aggregation cases. 
We introduce corrections as a function of temperature for the standard transition free energy between different phases to reproduce the phase boundaries over a range of temperatures. For instance, we found that as for the lamellar-cylindrical phase transition of sodium palmitate at $86^{\circ} \mathrm{C}$, the values of $\delta \mu_{a m p}^{0,2}=\Delta \mu_{a m p}^{0, E}-\Delta \mu_{a m p}^{0, D}=0.920 k T$ and $\delta \mu_{a m p}^{0,3}=\Delta \mu_{a m p}^{0, L_{1}}-\Delta \mu_{a m p}^{0, E}=-0.005 k T$ reproduce the boundary at $63.1 \%-49.1 \%$ (weight percent) in comparison with experimental observation $63 \%-49 \%$; while for the cylindricalmicellar transition, the values of $\delta \mu_{m o n}^{0,3}=1.594 k T$ and $\delta \mu_{w a t}^{0,2}=-0.005 k T$ produces boundary at $34.2 \%-25.8 \%$ comparing to experimental observation $33.8 \%-26.5 \%$. It is convenient to represent chemical potentials of surfactant and water molecules as shown in the left panel of Fig.3. As the amphiphilic molecule concentration increases, the water molecule chemical potential drops and the amphiphilic molecule chemical potential increases. When the concentration is low, the micelle aggregates dominate. As the concentration increases, the cylindrical aggregate appears. The intersection of the micelle curve with the cylinder curve represents the equilibrium between the two phases. When the concentration goes higher, cylindrical aggregate begins to dominate till the lamelle aggregate starts to form at the intersection of the cylinder curve with the lamelle curve. At higher concentrations, there is only lamelle aggregates. Comparison with the experimental aggregate size $b$ in both the lamellar and the cylindrical phases are shown in the right panel of Fig.3. A good agreement is obtained. For both lamellar and cylindrical phase, along with the increase of amphiphilic molecule concentration, the aqueous bulk size $L$ decreases and the aggregate size $b$ increases till the extended aggregate size $\mathrm{b}_{\text {exd }}=20.93 \AA$ is reached in the lamellar phase.

Prior to calculating the binary phase diagram for the carboxylate soap-water system, we have to understand how the model parameters (such as specific volume, intrinsic surface tension $\gamma_{0}$, spontaneous curvature $H_{0}$, as well as the standard transition chemical potential $\left.\delta \mu_{\text {mon }}^{0,2}, \delta \mu_{\text {mon }}^{0,3}\right)$ vary with temperature. We interpolated the specific volume of $\mathrm{C} 14 \mathrm{Na}$ at $86^{\circ} \mathrm{C}$ as $1.022(\mathrm{~mL} / \mathrm{g})$ from the other sodium carboxylate soaps as listed in Table.3 (Appendix C). 

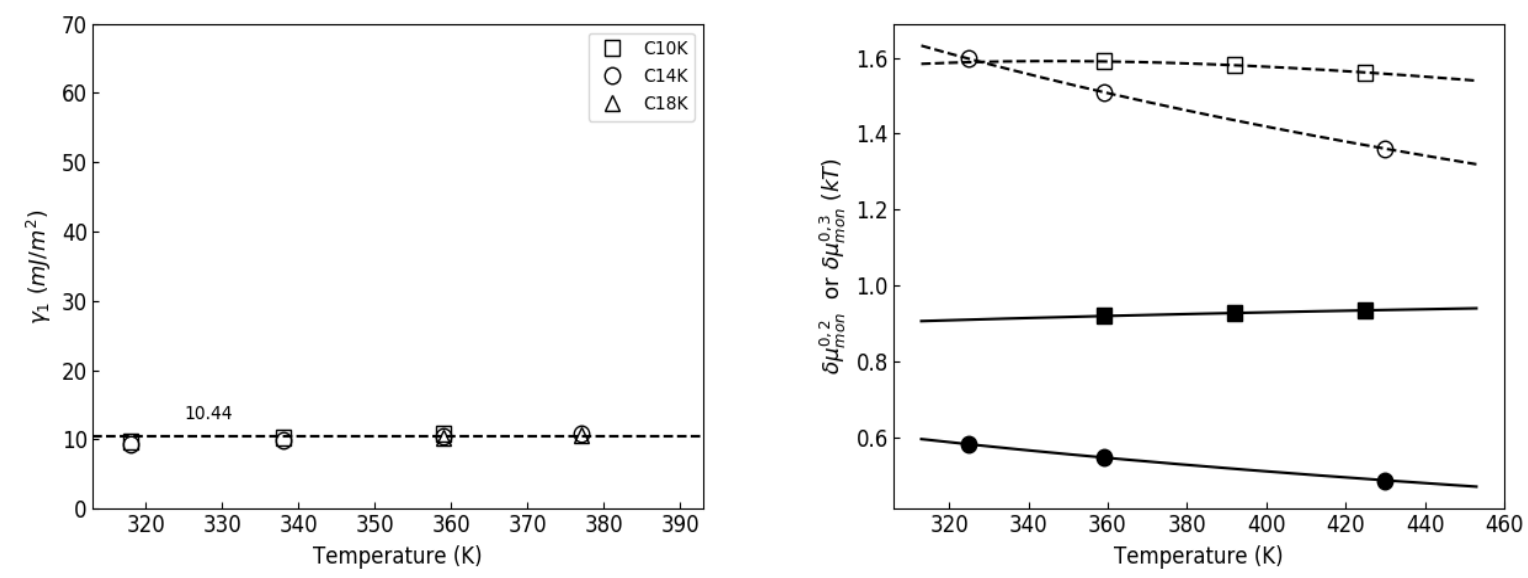

Figure 4: Left Panel, the surface tension $\gamma_{1}$ of lamellar aggregates. squares are for C10K , circles for $\mathrm{C} 14 \mathrm{~K}$ and up-triangles for C18K. Right panel, the dependence of the standard transition chemical potentials of palmitate soaps on the temperature. The square is for $\mathrm{Na}^{+}$and circle for $\mathrm{K}^{+}$. The open symbols are for the monomer transition free energy from lamellar phase to cylindrical phase $\delta \mu_{\text {mon }}^{0,2}$ and solid symbols are that from cylindrical phase to micellar phase $\delta \mu_{\text {mon }}^{0,3}$. The lines are fitting results.

The temperature dependence of specific volume of $\mathrm{C} 16 \mathrm{Na}$ is

$$
s v=24.55587 /(T+273)+0.001169(T+273)+0.553052
$$

and we list the coefficients of all the other carboxylate soaps specific volume in Table.4 (Appendix C)

In the left panel of Fig. 4 we shows that the surface tension $\gamma_{1}$ variation over temperature. The lamellar aggregation dimensions are measured for potassium carboxylate soaps at three more temperatures, $45^{\circ} \mathrm{C}, 65^{\circ} \mathrm{C}$ and $104^{\circ} \mathrm{C}$. Using the same method above, we calculated the $\gamma_{1}$ values for $\mathrm{C} 10 \mathrm{~K}, \mathrm{C} 14 \mathrm{~K}$ and $\mathrm{C} 18 \mathrm{~K}$ over three temperatures. It is observed that the surface tension is nearly independent of the temperatures with deviation of $5.12 \%$ away from the $10.44 \mathrm{~mJ} / \mathrm{m}^{2}$. This confirms the statements by Wennerstrom ${ }^{13}$ and Parsegian ${ }^{31}$ that the $\gamma_{1}$ depends only slightly on temperature. And hence we assumed that both the intrinsic surface tension $\gamma_{0}$ and spontaneous curvature $H_{0}$ also depend slightly on temperature since the sum $\gamma_{1}=\gamma_{0}+2 \kappa H_{0}^{2}$ are nearly independent on temperature and the length of tail-group. It turns 
out that the intrinsic surface tension $\gamma_{0}$, spontaneous curvature $H_{0}$ and blending constant $\kappa$ can be roughly regarded as a constant for an aggregate with a particular counter-ion.
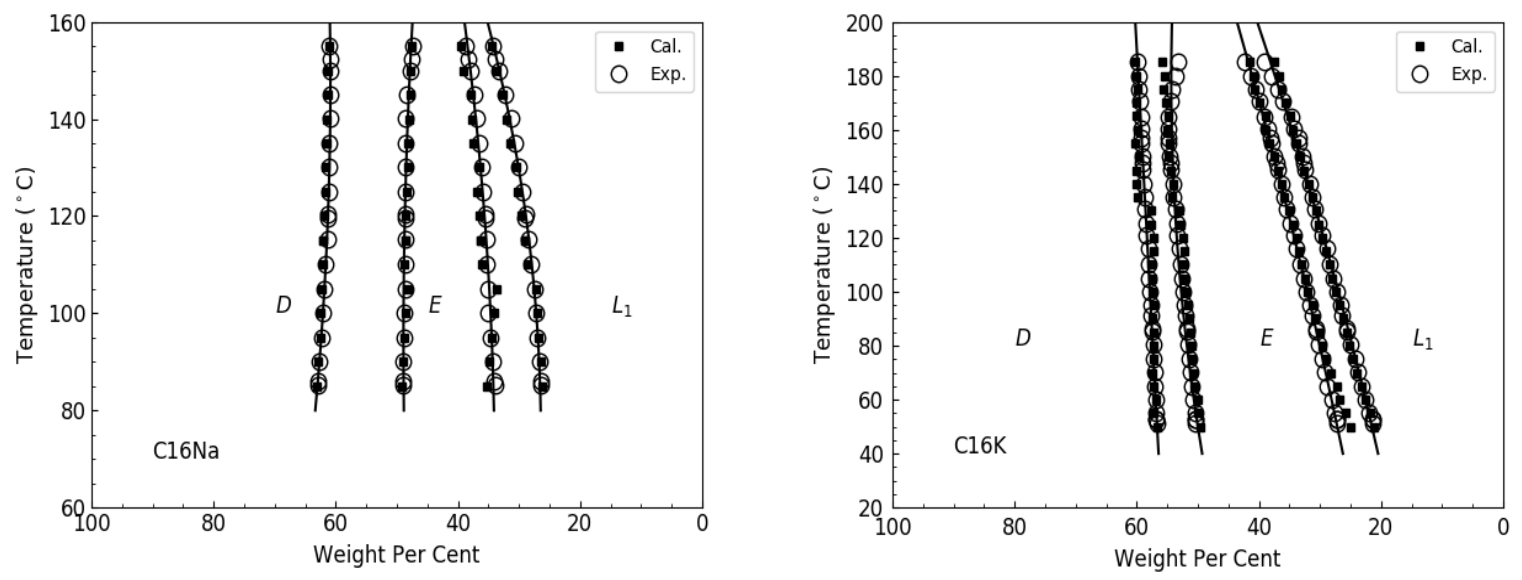

Figure 5: Left Panel, the Sodium Palmitate phase diagram. Right Panel, the Potassium Palmitate phase diagram. The $\mathrm{D}$ is for the lammellar aggregation, $\mathrm{E}$ for the cylindrical aggregation, $\mathrm{L}_{1}$ for the micellar aggregation. The solid lines are the 2 nd-order polynomial fitting results.

The variations of standard transition chemical potential $\left(\delta \mu_{m o n}^{0,2}\right.$ and $\left.\delta \mu_{\text {mon }}^{0,3}\right)$ along with temperature are shown in the right panel of Fig.4. We calculated the phase boundaries at three temperatures for both potassium carboxylate soaps and sodium carboxylate soaps. By adjusting the standard transition chemical potentials for monomer and water molecules, we reproduced the experimental observations of the phase boundaries for both sodium soaps ${ }^{35}$ and potassium soaps. ${ }^{36}$ We obtained the empirical temperature dependence for carboxylate soap systems, such as for $\mathrm{C} 16 \mathrm{Na}$

$$
\begin{aligned}
& \left(\delta \mu_{a m p}^{0,2}\right) / k T=-27.4606979 /(T+273)-0.000047(T+273)+0.9785150 \\
& \left(\delta \mu_{a m p}^{0,3}\right) / k T=-247.1463 /(T+273)-0.002059(T+273)+3.017693
\end{aligned}
$$

The chemical potential corrections of $\mathrm{C} 18 \mathrm{Na}$ for water molecules are shown in in the Fig.7 in Appendix C. The step-feature of them over temperature indicates that the fitting with function above is rahter crude. All the coefficients for surfactant and water correction functions are listed in Table 5 and Table 6 in the Appendix D. 
Once all the parameters temperature dependence are established (note that the dielectric constant temperature dependence is rather crude since the limited experimental measurement we could find for carboxylate soap solution at various temperatures. This definitely contributes to the corrections above), the excess chemical potentials of surfactant and water molecule are determined for three aggregate geometries at optimum aggregate sizes and over a range of temperatures. Good agreements with experimentally determined phase boundaries are shown in Fig.5 except at higher (near to the critical temperatures), indicating that the continuum model describes the main features quantitatively over range of nearly $100^{\circ} \mathrm{C}$. The calculated phase diagram for other carboxylate soaps are shown in Fig.8 in the Appendix D.

\section{Conclusion}

The continuum model has been build-up from cell model by incorporating the elastic surface free energy and dispersion interactions between counter-ions and the charged aggregate surface. The Helfrich free energy is applied to the aggregate surface energy and both the intrinsic surface tension $\gamma_{0}$ and spontaneous curvature $H_{0}$ as well as the surface tension $\gamma_{1}$ are calculated through the Gob optimization ( the aggregation dimension sizes are observed in thermodynamic measurements) by using the differential evolution algorithm. When the dispersion interaction is incorporated into the Poisson-Boltzmann equation, the distributions of the counter-ions in the aqueous region exhibit significant ion-specific behavior, where we can see that more $\mathrm{K}^{+}$are attached to the aggregate surface than $\mathrm{Na}^{+}$does. That the $\mathrm{K}^{+}$reduces more reside contact of the apolar tail of amphiphilic molecules with waters than the $\mathrm{Na}^{+}$ does results in the surface tension $\gamma_{1}$ for the potassium carboxylate lamelles are much less than that of the sodium carboxylate lamelles. The same observation has been shown for the cylindrical aggregations. The calculations of continuum model over different temperatures and over different carboxylate soaps show us that both lamellar aggregate surface tension $\gamma_{1}$ 
the intrinsic surface tension $\gamma_{0}$, spontaneous curvature $H_{0}$ and blending constant $\kappa$ are approximately independent of temperature and carbon number of the tail groups. This allows for the binary phase boundaries calculations for carboxylate soap solutions over the a range of temperatures. Good quantitative descriptions of aggregate behaviour over the compositions have been made by introducing standard transition chemical potentials for amphiphile monomer and water molecule. Since the continuum model reproduces main features of the carboxylate soaps aggregation, further development of this model allows unraveling more interesting physics in the self-assembly of amphiphilic molecules.

\section{References}

(1) Tanford, C. Micelle shape and size. The Journal of Physical Chemistry 1972, 76, 30203024.

(2) Tanford, C. Theory of micelle formation in aqueous solutions. The Journal of Physical Chemistry 1974, 78, 2469-2479.

(3) Gunnarsson, G.; Joensson, B.; Wennerstroem, H. Surfactant association into micelles. An electrostatic approach. The Journal of Physical Chemistry 1980, 84, 3114-3121.

(4) Helfrich, W. Elastic properties of lipid bilayers: theory and possible experiments. Zeitschrift für Naturforschung C 1973, 28C, 693-703.

(5) De Gennes, P. G.; Taupin, C. Microemulsions and the flexibility of oil/water interfaces. The Journal of Physical Chemistry 1982, 86, 2294-2304.

(6) Wennerstrom Hakan, O. U. Microemulsions as model systems. Comptes Rendus Chimie 2009, 12, 4-17.

(7) Boström, M.; Williams, D. R. M.; Ninham, B. W. Specific Ion Effects: Why DLVO Theory Fails for Biology and Colloid Systems. Phys. Rev. Lett. 2001, 87, 168103. 
(8) Boström, M.; Williams, D. R. M.; Ninham, B. W. Surface Tension of Electrolytes: Specific Ion Effects Explained by Dispersion Forces. Langmuir 2001, 17, 4475-4478.

(9) Boström, M.; Williams, D. R. M.; Ninham, B. W. Ion Specificity of Micelles Explained by Ionic Dispersion Forces. Langmuir 2002, 18, 6010-6014.

(10) Lukanov, B.; Firoozabadi, A. Specific Ion Effects on the Self-Assembly of Ionic Surfactants: A Molecular Thermodynamic Theory of Micellization with Dispersion Forces. Langmuir 2014, 30, 6373-6383, PMID: 24832546.

(11) Jönsson, B.; Nilsson, P.-G.; Lindman, B.; Guldbrand, L.; Wennerström, H. In Surfactants in Solution; Mittal, K. L., Lindman, B., Eds.; Springer US: Boston, MA, 1984; pp 3-21.

(12) Jönsson, B.; Wennerstrom, H. Thermodynamics of ionic amphiphile - water systems. Journal of Colloid and Interface Science 1981, 80, 482 - 496.

(13) Jönsson, B.; Gunnarsson, G.; Wennerström, H. In Solution Behavior of Surfactants: Theoretical and Applied Aspects Volume 1; Mittal, K. L., Fendler, E. J., Eds.; Springer US: Boston, MA, 1982; pp 317-341.

(14) A.L.Loeb, J.; P.H.Wiersema, The Electrical Double Layer Around a Spherical Colloid Particle; M.I.T. Press, Cambridge, 1961.

(15) Stigter, D. Functional representation of properties of the electrical double layer around a spherical colloid particle. Journal of Electroanalytical Chemistry and Interfacial Electrochemistry 1972, 37, $61-64$.

(16) Stigter, D. Micelle formation by ionic surfactants. I. Two phase model, Gouy-Chapman model, hydrophobic interactions. Journal of Colloid and Interface Science 1974, 47, 473 - 482, Proceedings of the 47th National Colloid Symposium ACS Division of Colloid and Surface Chemistry. 
(17) Gruen, D. W. R. A model for the chains in amphiphilic aggregates. 1. Comparison with a molecular dynamics simulation of a bilayer. The Journal of Physical Chemistry 1985, $89,146-153$.

(18) Gruen, D. W. R. A model for the chains in amphiphilic aggregates. 2. Thermodynamic and experimental comparisons for aggregates of different shape and size. The Journal of Physical Chemistry 1985, 89, 153-163.

(19) Ben-Shaul, A.; Szleifer, I.; Gelbart, W. M. Statistical thermodynamics of amphiphile chains in micelles. Proceedings of the National Academy of Sciences 1984, 81, 46014605 .

(20) Ben-Shaul, A.; Szleifer, I.; Gelbart, W. M. Chain organization and thermodynamics in micelles and bilayers. I. Theory. The Journal of Chemical Physics 1985, 83, 3597-3611.

(21) Szleifer, I.; Ben-Shaul, A.; Gelbart, W. M. Chain organization and thermodynamics in micelles and bilayers. II. Model calculations. The Journal of Chemical Physics 1985, 83, 3612-3620.

(22) Shaul, B. A.; Gelbart, W. M. Theory of Chain Packing in Amphiphilic Aggregates. Annual Review of Physical Chemistry 1985, 36, 179-211.

(23) Nagarajan, R.; Ruckenstein, E. Theory of surfactant self-assembly: a predictive molecular thermodynamic approach. Langmuir 1991, 7, 2934-2969.

(24) Camesano, T. A.; Nagarajan, R. Micelle formation and CMC of gemini surfactants: a thermodynamic model. Colloids and Surfaces A: Physicochemical and Engineering Aspects 2000, 16\%, $165-177$.

(25) Storn, R.; Price, K. Differential Evolution - A Simple and Efficient Heuristic for global Optimization over Continuous Spaces. Journal of Global Optimization 1997, 11, 341359. 
(26) Nagarajan, R. Molecular Packing Parameter and Surfactant Self-Assembly: The Neglected Role of the Surfactant Tail. Langmuir 2002, 18, 31-38.

(27) Gallot, B.; Skoulios, A. Interactions électriques dans les phases mésomorphes des systèmes amphiphile-eau: Rôle de la teneur en eau, de la longueur de la chaîne paraffinique, de la nature du cation, et de la température. Kolloid-Zeitschrift und Zeitschrift für Polymere 1966, 208, 37-43.

(28) Skoulios, A. La structure des solutions aqueuses concentrées de savon. Advances in Colloid and Interface Science 1967, 1, 79 - 110.

(29) Ninham, B.; Nostro, P. MOLECULAR FORCES AND SELF ASSEMBLY; CAMBRIDGE UNIVERSITY PRESS: New York, 2010.

(30) Parsons, D. F.; Ninham, B. W. Ab Initio Molar Volumes and Gaussian Radii. The Journal of Physical Chemistry A 2009, 113, 1141-1150, PMID: 19140766.

(31) Parsegian, V. A. Theory of liquid-crystal phase transitions in lipid + water systems. Trans. Faraday Soc. 1966, 62, 848-860.

(32) Blackburn, J. C.; Kilpatrick, P. K. Electrostatic Modeling of Surfactant LiquidCrystalline Aggregates: The Modified PoissonBoltzmann Equation. Industrial \& Engineering Chemistry Research 1996, 35, 2823-2833.

(33) Yuet, P. K.; Blankschtein, D. Molecular-Thermodynamic Modeling of Mixed Cationic/Anionic Vesicles. Langmuir 1996, 12, 3802-3818, PMID: 27682332.

(34) Tanford, C. The hydrophobic effect; Wiley, New York, 1973.

(35) McBain, J. W.; Lee, W. W. Vapor pressure data and phase diagrams for some concentrated soap-water systems above room temperatures. Oil and Soap 1943, 20, 17-25. 
(36) McBain, J. W.; Sierichs, W. C. The solubility of sodium and potassium soaps and the phase diagrams of aqueous potassium soaps. Journal of the American Oil Chemists' Society 25, 221-225. 


\section{Appendix}

\section{Appendix A}

$$
\begin{aligned}
E_{e l}+E_{d i s}-T S_{m i x} & =\frac{1}{2} n_{m o n}^{a}(-e) \phi_{A}+\frac{F f_{d}}{2} \int_{b}^{b+L}\left(c_{i o n}-c_{m o n}\right) \phi(r) r^{d-1} d r+N_{A} f_{d} \int_{b}^{b+L} c_{i o n} U_{d i s p}(r) r^{d-1} d r \\
& +R T f_{d} \int_{b}^{b+L}\left\{c_{i o n}\left[\ln \left(c_{i o n} / c_{0}\right)-1\right]+c_{m o n}\left[\ln \left(c_{m o n} / c_{0}\right)-1\right]\right\} r^{d-1} d r
\end{aligned}
$$

and then

get

$$
\begin{aligned}
E_{e l}+E_{\text {dis }}-T S_{\text {mix }} & =\frac{1}{2} n_{\text {mon }}^{a}(-e) \phi_{A}+\frac{R T f_{d}}{2} \int_{b}^{b+L} \frac{\left(c_{\text {ion }}-c_{\text {mon }}\right) e \phi(r)}{k T} r^{d-1} d r \\
& +N_{A} f_{d} \int_{b}^{b+L} c_{\text {ion }} U_{d i s p}(r) r^{d-1} d r \\
& +R T f_{d} \int_{b}^{b+L}\left\{\left[c_{\text {ion }} \ln \left(c_{\text {ion }}^{0} / c_{0}\right)-\frac{c_{\text {ion }} e \phi(r)}{k T}-\frac{c_{\text {ion }} U_{\text {disp }}(r)}{k T}-c_{\text {ion }}\right]\right. \\
& \left.+\left[c_{\text {mon }} \ln \left(c_{\text {mon }}^{0} / c_{0}\right)+\frac{c_{\text {mon }} e \phi(r)}{k T}-c_{\text {mon }}\right]\right\} r^{d-1} d r \\
& =n_{\text {mon }}^{a}(-e) \phi_{A}-E_{\text {el }}+k T\left[n_{\text {ion }}^{w} \ln \left(c_{\text {ion }}^{0} / c_{0}\right)+n_{\text {mon }}^{w} \ln \left(c_{\text {mon }}^{0} / c_{0}\right)\right]-k T\left(n_{\text {ion }}^{w}+n_{\text {mon }}^{w}\right)
\end{aligned}
$$




\section{Appendix B}
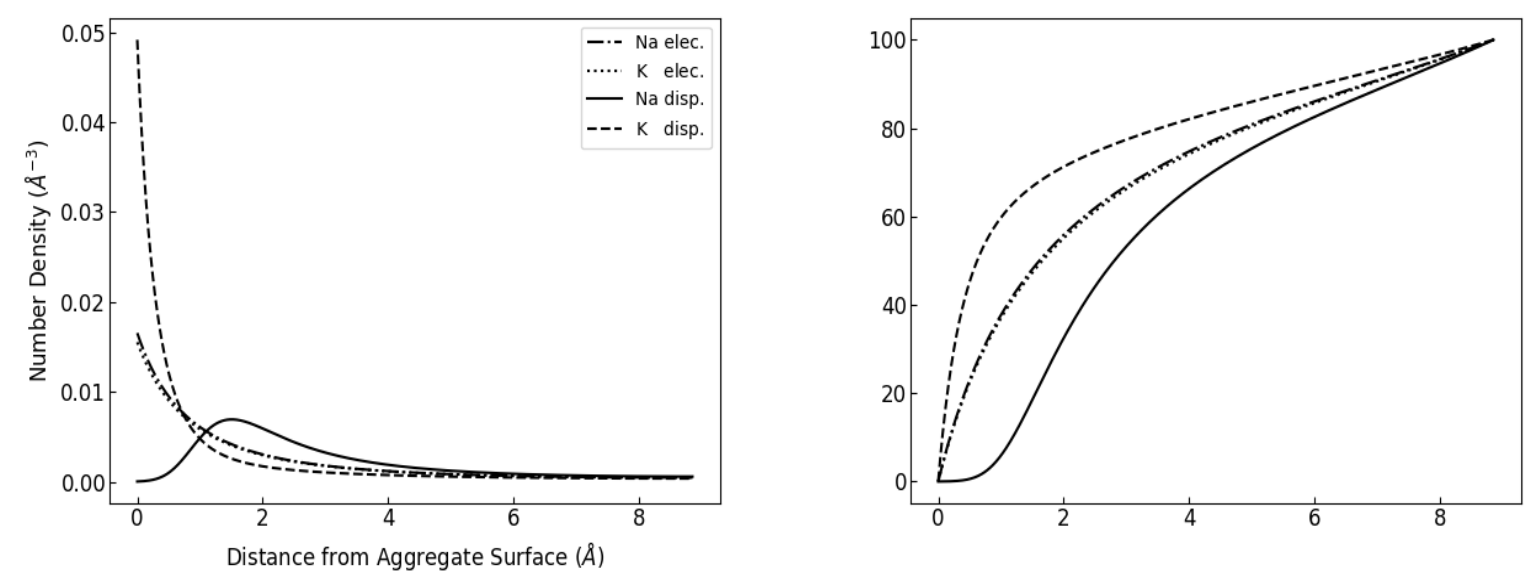

Figure 6: Left Panel, the counter-ion number density in the aqueous region of cylindrical aggregations. The aqueous region size $L=8.86 \AA$. For electrostatic system, aggregate size $b=18.13 \AA$ for the potassium palmitate and $b=18.14 \AA$ for the sodium palmitate; For the dispersion system, $\mathrm{b}=18.16 \AA$ for both soaps. Right panel, the fraction of the counter-ion attached to the cylindrical aggregate surface. For both plots, the dash-dotted and dotted lines are for the $\mathrm{Na}^{+}$and $\mathrm{K}^{+}$only with electrostatic interaction, respectively; The solid and dashed lines are for the $\mathrm{Na}^{+}$and $\mathrm{K}^{+}$with dispersion, respectively.

Table 2: Cylindrical Aggregation Spontaneous Curvature $H_{0}\left(\AA^{-1}\right)$ and Bending Constant $\kappa\left(\mathrm{mJ} / \mathrm{m}^{2} \AA^{2}\right)$

\begin{tabular}{lllllllll}
\hline $\mathrm{Na}^{+}$Elect. & 8 & 10 & 12 & 14 & 16 & 18 & 20 & 22 \\
$\mathrm{H}_{0}$ & & & 0.0291 & & 0.0231 & 0.0201 & & \\
$\kappa$ & & & 5027.9 & & 6808.9 & 4665.7 & & \\
\hline $\mathrm{K}^{+}$Elect. & & & & & & & & \\
$\mathrm{H}_{0}$ & 0.0394 & 0.0373 & 0.0290 & 0.0272 & 0.0241 & 0.0219 & 0.0210 & 0.0216 \\
$\kappa$ & 3297.0 & 2416.3 & 5135.3 & 4267.6 & 5568.5 & 6406.2 & 5436.4 & 4044.1 \\
\hline \hline $\mathrm{Na}^{+}$Disp. & \multicolumn{1}{c}{0.0383} & & 0.0335 & 0.0320 & & \\
$\mathrm{H}_{0}$ & & & 0.0373 .4 & & 6312.2 & 5624.4 & & \\
$\kappa$ & & & 5273.1 & & \\
\hline $\mathrm{K}^{+}$Disp. & & & & & & & & \\
$\mathrm{H}_{0}$ & 0.0394 & 0.0364 & 0.0282 & 0.0257 & 0.0229 & 0.0206 & 0.0192 & 0.0186 \\
$\kappa$ & 1714.8 & 1456.7 & 3482.0 & 3357.4 & 4323.2 & 5329.1 & 4994.6 & 4211.1 \\
\hline
\end{tabular}




\section{Appendix C}

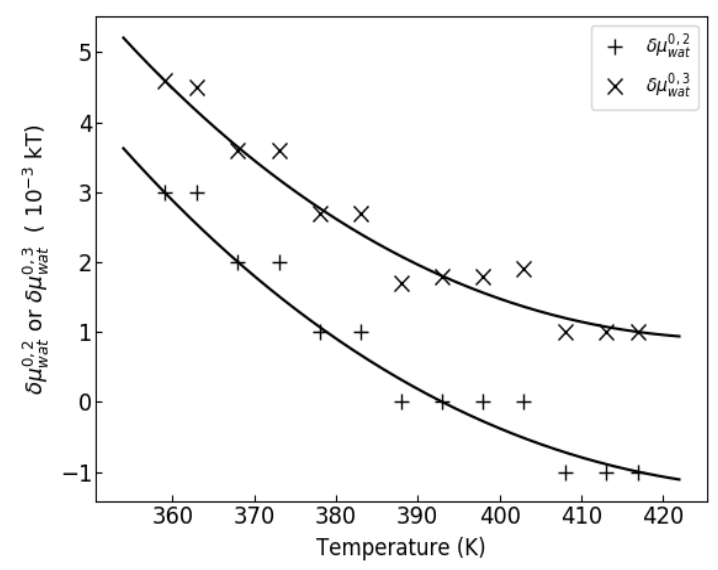

Figure 7: The water standard transition chemical potential variation over temperatures for C18Na. The + is for $\delta \mu_{w a t}^{0,2}$ and the $\times$ is for the $\delta \mu_{w a t}^{0,3}$.

Table 3: Carboxyle soap specifc volume $(\mathrm{ml} / \mathrm{g})$

\begin{tabular}{lllllllll}
\hline & 8 & 10 & 12 & 14 & 16 & 18 & 20 & 22 \\
$\mathrm{Na}^{+}$ & 0.936 & & 1.002 & & 1.041 & 1.055 & & \\
$\mathrm{~K}^{+}$ & 0.904 & 0.943 & 0.973 & 0.996 & 1.015 & 1.031 & 1.044 & 1.055 \\
\hline
\end{tabular}

Table 4: Carboxyle soap specifc volume dependence on Temperature Dependence coefficients, $\mathrm{a} /(\mathrm{T}+273)+\mathrm{b}(\mathrm{T}+273)+\mathrm{c}, \mathrm{a}=24.55587, \mathrm{~b}=0.001169$, all $\mathrm{c}$ values

\begin{tabular}{lllll}
\hline & 12 & 14 & 16 & 18 \\
$\mathrm{Na}^{+}$ & 0.514052 & 0.534052 & 0.553052 & 0.567052 \\
$\mathrm{~K}^{+}$ & 0.485052 & 0.508100 & 0.527052 & 0.543052 \\
\hline
\end{tabular}




\section{Appendix D}

Table 5: Sodium carboxylate monomer and water standard transition chemical potential dependence on Temperature, a/(T+273) + b $(\mathrm{T}+273)+\mathrm{c}, \mathrm{T}\left({ }^{\circ} \mathrm{C}\right)$

\begin{tabular}{llll}
\hline $\mathrm{C} 12 \mathrm{Na}$ & $\mathrm{a}$ & $\mathrm{b}$ & $\mathrm{c}$ \\
$\delta \mu_{a m p}^{0,2}$ & 35.49129 & 0.000038 & 0.9384150 \\
$\delta \mu_{a m p}^{0,3}$ & 298.7184 & 0.001488 & 0.5565790 \\
$\delta \mu_{w a t}^{0,2}$ & 0.000000 & 0.000000 & $15.000000(\mathrm{~T}<85)$ \\
& -78869.54 & -0.640502 & $464.63265(\mathrm{~T} \geq 85)$ \\
$\delta \mu_{w a t}^{0,3}$ & 0.000000 & 0.000000 & $22.000000(\mathrm{~T}<85)$ \\
& -95629.32 & -0.714109 & $547.74209(\mathrm{~T} \geq 85)$ \\
\hline & & & \\
$\mathrm{C} 14 \mathrm{Na}$ & $\mathrm{a}$ & $\mathrm{b}$ & $\mathrm{c}$ \\
$\delta \mu_{a m p}^{0,2}$ & 263.7701 & 0.001616 & -0.2947240 \\
$\delta \mu_{a m p}^{0,3}$ & -1027.604 & -0.007648 & 7.3730930 \\
$\delta \mu_{w a t}^{0,2}$ & 0.000000 & 0.000000 & 8.0000000 \\
$\delta \mu_{w a t}^{0,3}$ & 0.000000 & 0.000000 & $12.000000(\mathrm{~T}<110)$ \\
& 0.000000 & 0.000000 & $-10.848000(\mathrm{~T} \geq 110)$ \\
\hline & & & \\
$\mathrm{C} 16 \mathrm{Na}$ & $\mathrm{a}$ & $\mathrm{b}$ & $\mathrm{c}$ \\
$\delta \mu_{a m p}^{0,2}$ & -27.46070 & -0.000047 & 0.9785150 \\
$\delta \mu_{a m p}^{0,3}$ & -247.1463 & -0.002059 & 3.0176930 \\
$\delta \mu_{w a t}^{0,2}$ & 0.000000 & 0.000000 & $-4.0000000(\mathrm{~T}<105)$ \\
& 0.000000 & 0.000000 & $-5.0000000(\mathrm{~T} \geq 105)$ \\
$\delta \mu_{w a t}^{0,3}$ & -27.46070 & -0.134527 & 119.78740 \\
\hline & & & \\
$\mathrm{C} 18 \mathrm{Na}$ & $\mathrm{a}$ & $\mathrm{b}$ & $\mathrm{c}$ \\
$\delta \mu_{a m p}^{0,2}$ & 256.0779 & 0.0015210 & -0.2793180 \\
$\delta \mu_{a m p}^{0,3}$ & -106.9063 & -0.0011110 & 2.2145210 \\
$\delta \mu_{w a t}^{0,2}$ & 47237.673 & 0.2465771 & 217.10243 \\
$\delta \mu_{w a t}^{0,3}$ & 49723.867 & 0.2700811 & -230.86572 \\
\hline & & & \\
& & \\
& &
\end{tabular}


Table 6: Potassium carboxylate monomer and water standard transition chemical potential dependence on Temperature, a/(T+273) + b $(\mathrm{T}+273)+\mathrm{c}, \mathrm{T}\left({ }^{\circ} \mathrm{C}\right)$

\begin{tabular}{llll}
\hline & & & \\
$\mathrm{C} 12 \mathrm{~K}$ & $\mathrm{a}$ & $\mathrm{b}$ & $\mathrm{c}$ \\
$\delta \mu_{a m p}^{0,2}$ & 70.781070 & -0.000301 & 0.4309120 \\
$\delta \mu_{a m p}^{0,3}$ & 39.226910 & -0.001684 & 2.1324510 \\
$\delta \mu_{w a t}^{0,2}$ & -2465.5800 & -0.041988 & 23.959083 \\
$\delta \mu_{w a t}^{0,3}$ & 9834.5740 & 0.049674 & -40.215934 \\
\hline & & & \\
$\mathrm{C} 14 \mathrm{~K}$ & $\mathrm{a}$ & $\mathrm{b}$ & $\mathrm{c}$ \\
$\delta \mu_{a m p}^{0,2}$ & 59.622130 & -0.000405 & 0.517162 \\
$\delta \mu_{a m p}^{0,3}$ & 190.40270 & -0.000771 & 1.291490 \\
$\delta \mu_{w a t}^{0,2}$ & 754.71050 & -0.017779 & 6.280536 \\
$\delta \mu_{w a t}^{0,3}$ & 754.71050 & -0.017779 & 7.280536 \\
\hline & & & \\
$\mathrm{C} 16 \mathrm{~K}$ & $\mathrm{a}$ & $\mathrm{b}$ & $\mathrm{c}$ \\
$\delta \mu_{a m p}^{0,2}$ & 88.080490 & -0.000274 & 0.399192 \\
$\delta \mu_{a m p}^{0,3}$ & 233.95760 & -0.000583 & 1.066614 \\
$\delta \mu_{w a t}^{0,2}$ & 0.0000000 & 0.000000 & 2.000000 \\
$\delta \mu_{w a t}^{0,3}$ & 0.0000000 & 0.000000 & $3.000000(\mathrm{~T}<155)$ \\
& 0.0000000 & 0.000000 & $2.000000(\mathrm{~T} \geq 155)$ \\
\hline & & & \\
$\mathrm{C} 18 \mathrm{~K}$ & $\mathrm{a}$ & $\mathrm{b}$ & $\mathrm{c}$ \\
$\delta \mu_{a m p}^{0,2}$ & 69.456590 & -0.000498 & 0.527468 \\
$\delta \mu_{a m p}^{0,3}$ & -10.115040 & -0.002520 & 2.442728 \\
$\delta \mu_{w a t}^{0,2}$ & 10115.040 & 0.019645 & -34.22800 \\
$\delta \mu_{w a t}^{0,3}$ & 12744.950 & 0.034752 & -46.87728 \\
\hline & & &
\end{tabular}



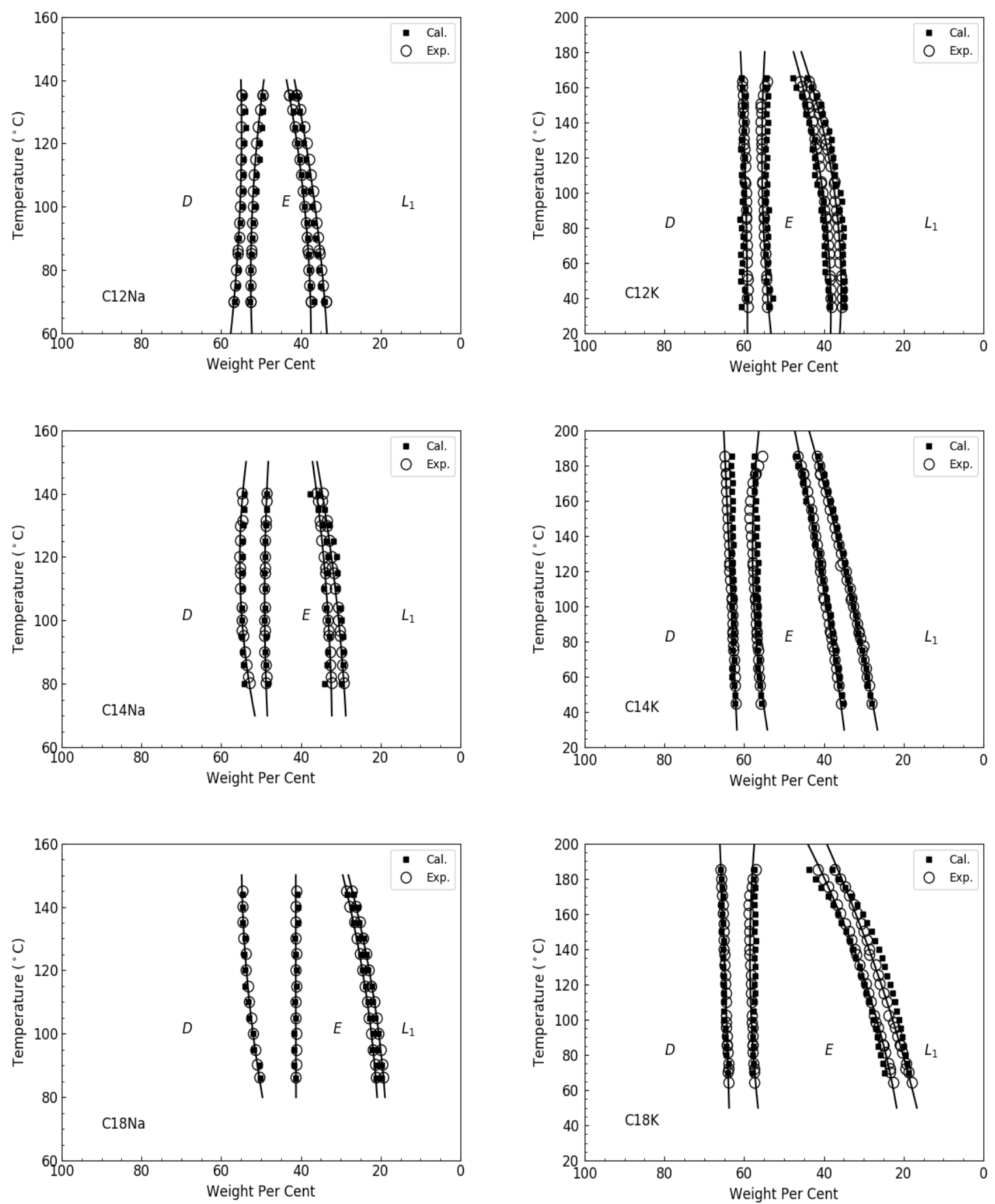

Figure 8: Left Panels, the phase diagrams for the C12Na, C14Na and C18Na. Right Panels, the phase diagrams $\mathrm{C} 12 \mathrm{~K}, \mathrm{C} 14 \mathrm{~K}$ and $\mathrm{C} 18 \mathrm{~K}$. The D is for the lammellar aggregation, $\mathrm{E}$ for the cylindrical aggregation, $\mathrm{L}_{1}$ for the micellar aggregation. The solid lines are the 2nd-order polynomial fitting results. 